\title{
EDOUARD-ALFRED MARTEL ET LES CAVERNES DE LA BELGIQUE
}

\author{
Daniel André*
}

\begin{abstract}
(Edouard-Alfred Martel and the caves of Belgium) An exhaustive report on the activity of Martel in Belgium is here given. In particular some relevant passages of Martel's letters and papers are also included.
\end{abstract}

\section{INTRODUCTION}

L'on sait qu'Edouard-Alfred Martel, célèbre spéléologue européen né en France dans la région parisienne, s'était rendu en Belgique en mai 1888. Il n'était pas venu qu'en simple touriste dans ce tout petit pays qu'il devait déjà connaître depuis longtemps, lui qui voyageait dans toute l'Europe avec ses parents depuis 1864 . Il avait ses raisons secrètes.

Le jeune explorateur qu'il aspirait à devenir rêvait de s'illustrer dans un domaine alors bien peu développé en France: l'étude des cavernes; ceci après avoir connu quelques déboires en matière de préhistoire.

Martel était, depuis l'enfance, attiré par le noir et les concrétions des cavernes.Lors de vacances en famille dans la région française des Grands Causses, il avait visité quelques grottes, dont celle de Nabrigas (gisement de faune quaternaire).

En septembre 1884, outre l'inspection des vastes entrées du spectaculaire site géologique de Bramabiau (Massif de l'Aigoual), il avait pu s'introduire dans la grotte des Demoiselles (qui était déjà ouverte au tourisme) et la grande salle de la grotte de Dargilan qui avait été découverte trois années auparavant par un jeune berger. Ces palais de cristal l'avaient fait rêver.

A partir de 1885, Martel n'avait plus qu'une idée en tête: mettre sur pied une expédition dans les Causses axée sur l'exploration souterraine, pour se faire un nom dans la science.

Il disposa de plus de deux années et demi pour ce faire, pour réfléchir à l'acquisition ou la réalisation d'un matériel adapté. Il se documenta et s'aguerrit aux rudes dépenses physiques lors de difficiles courses en montagne.

Il avait fixé la date de sa première campagne souterraine à l'extrême fin du mois de juin 1888 .

*Président de l'Association “Edouard-Alfred Martel " Chemin de la Lèche F - 48320 ISPAGNAC (France). 
Probablement pour se donner du courage, du stimulant pour ce qui était alors un projet audacieux et potentiellement périlleux, il avait fait un " crochet", sur la route des vacances dans les Causses, par la Belgique, en mai 1888,dans le seul dessein d'aller admirer les splendeurs stalagmitiques et aquatiques des grottes de Han-surLesse et de Rochefort.

Ces deux cavernes allaient lui servir de référence pour ses premiers travaux, à Dargilan surtout, et à Bramabiau.

Une fois sa renommée internationale établie, Martel fut appelé par des géologues belges pour venir leur faire part de ses connaissances (conférences), ainsi que pour qu'il vienne explorer lui même les cavités les plus prestigieuses du royaume.

Sa précieuse collaboration fut recherchée par de nombreux savants belges, parfois éminents et officiels.

Son nom, le poids de ses travaux et sa notoriété, attirèrent tout spécialement deux explorateurs de cavernes belges : le grand géologue Ernest Van-den-Broeck et le vulgarisateur et archéologue Edmond Rahir. De leur spéciale collaboration devait résulter,outre plusieurs articles, un énorme ouvrage (publié en 1910 en deux tomes) qui est considéré comme la bible de la spéléologie de ce pays: Les cavernes et les rivières souterraines de la Belgique,fort de plus de 1750 pages !

De nombreux articles et ouvrages d'ordre biographique ont été consacrés à Martel, dont la vie et l'œuvre sont assez bien connues. Tout a été dit sur ses relations ave cles Irlandais, les Américains, les Anglais, les Espagnols, etc.

Mais rien, pratiquement,n'a été développé sur les contacts que Martel a eut avec ses homologues de la Belgique, alors même que, de tous les pays qu'il a étudiés à l'étranger, c'est celui où il a accompli une œuvre la plus structurée de toute sa carrière, mais non la plus parfaite, loin de là.

Le présent fascicule del'International Journal of Speleology consacré à Martel offre l'occasion de s'arrêter quelque peu sur l'œuvre spéléologique en Belgique du grandsavant, ceci grâce à la découverte toute récente d'un fabuleux lot d'archives chez les descendants de ses héritiers (familles Renouard et Legrand).

\section{QUI ÉTAITE DOUARD-ALFRED MARTEL, BIENFAITEUR DES GRANDS CAUSSES ET PIONNIER DE LA SPÉLÉOLOGIE BELGE?}

Edouard-Alfred Martel, né à Pontoise le 1er juillet 1859 et décédé à Saint-Thomasla-Garde(Loire) le 03 juin 1938, a fondé la spéléologie française méthodiquement organisée à partir de ses recherches souterraines commencées en Lozère dès 1884 (grottes de Dargilan et de Nabrigas).

Issu d'une famille de juristes aisés, voué lui même à une carrière d'avocat, Martel fut un brillant étudiant que ses goûts poussèrent à s'intéresser à la géographie à un point tel qu'en 1877 il décrocha dans cette matière le premier prix au Concours Général !

Remarqué tout jeune par d'éminents savants, dont le célèbre pyrénéiste Alphonse Lequeutre, celui-ci lui donna pour mission d'explorer la Lozère et les Grands 
Causses et d'en révéler les mille curiosités aux Français.

Dès 1883 il entra en scène,pour ne cesser son labeur de propagandiste en faveur de la Lozère, l'un des plus beaux départements de France, qu'en 1935.

Entre les deux dates, il explora ou visita plus d'un millier de cavités (certains auteurs affirment le chiffre, probablement exagéré, de 4000 incursions souterraines).

Quant aux explorations de gouffres,elles se succédèrent, sans véritable arrêt, de 1888 jusque, aumoins, en 1926, année où il descendra une dernière fois grâce à des agrès (à l'aven Armand).

Son œuvre est immense; elle touche à tant de domaines qu'il n'est pas possible de songer ici à la résumer.

Martel, outre qu'il fut le pionnier incontesté de la Spèlèologie d'exploration dans l'Europe tout entière, fut aussi l'initiateur du Club Cévenol, le propagandiste acharné des Gorges du Tatn, l'inspirateur des Parcs Nationaux Français, un bienfaiteur de l'Humanité (travaux sur la préservation de la qualité des eaux de consommation), et surtout, le grand artisan de l'industrie touristique du Plateau Central de la France. Par souscription publique auprès de la population reconnaissante des Causses et des Cévennes, fut érigé le 11 junin 1927 un monument à la gloire d'Edouard-Alfred Martel et de son précieux auxiliaire lozérien, le forgeron Louis Armand, inventeur du gouffre célèbre qui porte son nom depuis tout juste un siècle.

Martel partage avec quatre grands français (Foch, Clémenceau, Saint-Saëns et Mistral) l'honneur insigne d'avoir été statufiéde son vivant, en présence de toutes les autorités régionales et d'un ministre, et surtout acclamé par une foule d'anonymes venus, de divers horizons, assister à son Apothéose.

On peut toujours voir ce monumentcommémoratif — vieux de soixante-douze années - au confluent du Tarn et de la Jonte, à l'une des extrémités du Pont de la Muze.

Martel y est "vivant": de son regard, toujours animé de cette foi qui lui a fait soulever les mystères de ces montagnes, il invite les centaines de milliers de visiteurs estivaux, qui honorent de leur passage notre belle nature, à mettre leur pas dans les siens...

Sa vie durant, Edouard-Alfred Martel fut acharné au travail, tant sous terre qu'à la surface, au coursd'explorations soutenues et riches en résultats. Il divulgua le fruit des études qui en découlèrent dans une quantité phénoménale d'articles ainsi que dan svingt-quatre ouvrages de librairie !

Un décompte non exhaustif fait état de 1008 titres distincts, chiffre qui, à lui seul, traduit bien son considérable labeur.

Martel fit paraître sa première publication en 1882 et sa dernière en 1936, soit cinquante-quatre années d'écriture qui ne connurent aucune césure...

L'ouvrage le plus connu de Martel es tson tout premier, qu'il titra "Les Cévennes" et qui connut, du vivant de son auteur, onze éditions successives ! Ce riche livre décrivait, en plus des serres et des vallées cévenoles, la Lozère tout entière, et surtout ses Grands Causses. Sa parution en décembre 1889 allait installer ce département sur une trajectoire touristique en perpétuelle expansion.

La plupart des autres ouvrages de Martel sont des sommes de connaissance, notamment son fameux et épais livre "Les Abîmes", paru en 1894 et consacré à la France souterraine connue à cette date, et, bien sûr, "Les cavernes et les rivières souterraines de la Belgique” qui est bien le pendant du précédent ouvrage. 
Le dernier cadeau que Martel fit à la Lozère fut la parution de ses "Causses Majeurs", en 1936. Edité à la fin de sa vie, au sommet de son art et de sa notoriété, cet ouvrage - véritable chant du cygne - est venu tel un testament transmettre aux caussenards l'héritage de la "terre des merveilles" qui était l'objet de ses constantes pensées, et qu'il leur incomberait désormais de faire fructifier .

Le département de la Lozère a tenu à honorer la mémoire de son grand bienfaiteur, en organisant en1997 l'Année Martel, qui fut un succès et qui fut l'occasion de l'édition de plusieurs ouvrages sur cet explorateur hors pair (ARCHIVES DEPARTEMENTALES et al 1999, ASSOCIATION E.-A. MARTEL 1997, FOUNTES 1997,PARC NATIONAL DES CEVENNES 1998).

\section{ETAT DES ARCHIVES MARTEL SUR LA BELGIQUE}

Cet article n'aurait puê tre rédigé tel quel sans la découverte d'un très riche dossier presque entièrement consacré aux cavernes belges,dossier que Martel avait précieusement conservé. Il n'a été retrouvé par Mme Nicole Legrand (petite-nièce de Martel) que tout récemment, par hasard.Celle-ci l'a transmis à Louis Renouard pour qu'il rejoigne l'énorme dépôt d'archives " martélienes " dont ce derniera, au sein de sa famille, la garde.

Louis Renouard, arrière petit-neveu de Martel et membre de l'Association Edouard-Alfred-Martel, l'a à son tour transmis à l'auteur de ces lignes pour étude.

Celle-ci sera bien longue, auvu de la masse impressionnante de documents (épistolaires surtout) qui ont été conservés. La finalité du présent article est d'en faire connaître l'existence aux chercheurs (au Belges tout spécialement), au moins sommairement, et en attendant mieux.

Nous avons sérié ainsi les documents :

1. Correspondance de 1889 à1910 ;

2. Plans originaux de cavernes de la Belgique ;

3. Articles manuscrits de Martel ;

4. Articles (typographiés) de Martel ;

5. Articles d'autres auteurs que Martel ;

6. Articles journalistiques surMartel et la Belgique ;

7. Photographies (cavernes et paysages belges);

1/ La correspondance totalise 246 lettres ou notes absolument inédites dont la publication, à elle seule, formerait un ouvrage historique sur la spéléologie belge. On relève 167 lettres d'Ernest Van-den-Broeck (souvent très copieuses), 35 lettres d'Edmond Rahir, 12 lettres ou notes de Martel, et 32 lettres de divers autres auteurs.

2/ Les plans originaux sont les suivants : schéma de la main d'Edmond Rahir (Courbes barométriques) ; fragment de carte géologique des environs de Bruxelles ; calque du plan original de la grotte de Rémouchamps pris sous terre par Martel (traces d'argile) ; calque du plan de la grotte de Dinant, par Rahir ; calques mis au propre à l'encre de chine, par Martel, des cavités suivantes : abîme de Comblain-auPont, caverne ossifère d'Engilhoul,grotte de Tilff, abîme de la Carrière Dantine à 
Ramioul, Trou du Diable à Ramioul, grotte du Trou-Maulin, résurgence de l'EauNoire, grotte du Pré-au-Tonneau près de Rochefort, siphon de Rémouchamps, Trou del'Abîme à Couvin, Trou des Nutons, Trou des Veaux, Trou Manto,Trou Méria près Celles, et grotte d'Epave; divers croquis sur la grotte de Han-sur-Lesse, plans de situation de cette même grotte (deux par Martel, un par Rahir et deux autres par Edouard Dupont), coupe développée (idem), quatre coupes transversales (idem); plan original sur papier millimétré de la grotte de Han-sur-Lesse, par Martel; divers tirages d'imprimerie de ces plans; plan en couleur d'un livret touristique (grotte de Hansur-Lesse) avec forces annotations de Martel et traces d'argile.

3/ Articles manuscrits. Quatre correspondent à des écrits publiés et parfaitement identifiables (voir, ci-dessous, références bibliographiques $n^{\circ} 6,8,11$ et 13 ). Un cinquième article comprend 43 feuilles écrites à la main par Martel (paginées de 40 à 83); il ne nous a pas été possible de le collationner avec aucun des écrits de Martel que possèdent séparément les membres de l'Association Martel (660 sur 1008 recensés). Plusieurs dates apparaissent dans ce texte dont la rédaction a visiblement été débutée vers 1928; la plus récente étant de 1938 ferait de ce texte le dernier de Martel (décédé cette année-là). A noter qu'il a dû se retrouver par hasard mêlé aux documents sur la Belgique.

4/ Trois articles typographiés, avec annotations de Martel (corrections, bons à tirer, etc.) ; il s'agit des textes suivants, de Martel : -1899- Onzième campagne souterraine de M. E.-A. Martel' in C. R. des Séances de la Société de Géographie, n4, séance d'avril, p.162-165; tiré à part Simon, Rennes, (janvier 1902), 4 p., titré Onzième campagne souterraine (1898), -1905- Les deux plus grandes cavernes d'Europe. Adelsberg et le Holl-Loch in Revue La Nature, ${ }^{\circ} 1676,8$ juillet 1905, p.90-91, -1910- chapitre "Les Abannets" de l'ouvrage “Les cavernes et les rivières souterraines de la Belgique".

5/ Divers articles d'autres auteurs que Martel. Un manuscrit sur des analyses d'eau de Modave (1890) dû au Dr E. Malloz ; quelques notes manuscrites, croquis imprimés, tirages typographiés avec corrections manuscrites de l'ouvrage" Les cavernes et rivières souterraines de la Belgique "; un long article manuscrit d'Ernest Van-den-Broeck, avec adjonctions de Martel, sur "les conditions de filtrage efficace des eaux souterraines dans certaines formations calcaires " (voir $\mathrm{n}^{\circ} 17$ de la bibliographie); un article de recension de Lucien Rudaux, sur "l'hydrologie souterraine de la Belgique ardennaise ", avec de très nombreuses corrections manuscrites de l'auteur lui même, de Martel et d'Ernest Van-den-Broeck ; un long texte tapuscrit de recension du gros ouvrage de 1910.

6/ Une soixantaine de coupures journalistiques, datant de 1898 à 1910 et traitant, pour la plupart, des venues de Martel en Belgique, de l'exploration de nouvelles cavités dans ce pays, et de comptes rendus bibliographiques relatifs à des productions de Martel et/ou collaborateurs belges. Ensemble très précieux pour bien caler la chronologie des faits et gestes de Martel en Belgique (voir bibliographie spéciale, plus bas).

7/ Des photographies, sur la Belgique et ses cavernes, dues majoritairement à Edmond Rahir et Ernest Van-den-Broek ; échantillons de roches ; sites karstiques de surface, dont deux avec Martel comme figurant; cavernes, pertes et paysages karstiques remis à Martel par Rahir; grotte de Han-sur-Lesse .

A noter qu'indépendamment des liasses d'archives conservés sur la Belgique par 
la famille Renouard-Legrand, un album photographi que consacré uniquement à ce pays (avec vues souterraines) a été cédé par Gérard Kalliatakis au Belge bien connu Guy de Block. Cet album avait appartenu à... Edouard-Alfred Martel lui-même (nombreux clichés de son cru).

La documentation historique de la Belgique souterraine est donc assez fournie.

\section{CHRONOLOGIE DES DÉPLACEMENTS DE MARTEL EN BELGIQUE. SITES, ET CAVITÉS VISITÉS ET/OU ÉTUDIÉS,CONFÉRENCES}

Martel a dit lui même quel fut le point de départ de ses recherches en Belgique, dans le grandouvrage qu'il a réalisé avec ses deux collaborateurs de ce pays.

“...Il m'est doux de rappeler, et le titre même du présent volume m'en inspire l'agréable devoir,qu'après une préliminaire et profonde impression de jeunesse à Adelsberg, dès 1879, c'est ma première visite de la grotte belge de Han-sur-Lesse, en 1888, qui, cette même année, me poussa sur le plan incliné des rivières souterraines et dans la verticale des abîmes. Plusieurs fois les circonstances et aussi la gratitude du souvenir, m'ont ramené depuis, non plus en touriste, mais en investigateur exercé, à Han, point de départ et cause efficiente de mes études préférées : en compagnie des deux amis qui ont élaboré ce livre avec moi, nous avons pu, dans cette admirable caverne, dégager, toute classique qu'elle soit depuis longtemps, certaines inconnues mystérieuses et corriger plusieurs fausses interprétations. Ce travail de mise au point, selon les données récentes de l'hydrologie souterraine particulièrement, s'est, tout naturellement et avec à propos, étendu par nos soins à tous trois, aux autres grottes de la Belgique".

Si Martel a probablement parcouru la Belgique dans toutes ses régions, il est patent qu'au point de vue spéléologique, invité en cela par ses collaborateurs du cru, il ne s'est vraiment intéressé qu'aux cavernes du Bassin carbonifère de Dinant (Lesse, Lomme, Wamme), dans le massif des Ardennes. Le Bassin de Namur (vaste région karstique de Liège et de Theux), qui renferme le Trou-Manto et la grotte de Spy, n'a été que survolé.

D'après nos recherches, Martel a au moins étudié 35 cavités ou émergences karstiques lors de ses séjours en Belgique, les plus importantes étant celles de Rochefort, Han-sur-Lesse et Rémouchamps.

D'après nos sources documentaires (longues recherches), Martel s'est au moins rendu dansce royaume dix neuf fois les années suivantes : 1888, 1890, 1898, 1900, 1901, 1902, 1903, 1905, 1908 et 1922.

La raison en est toute simple: de tous les massifs karstiques importants que pouvait étudier Martel, les calcaires de la Belgique sont les plus proches géographiquement de Paris. En peu de temps, il pouvait y aller par train.

Il a passé au minimum une quarantaine de jours en Belgique, de 1888 à 1922 (son plus long séjour a eu lieu en 1902, ce qui devait le décider à préparer le grand ouvrage).

Preuve de 1'attrait qu'exerçait chez lui ce pays, il a même failli réussir à s'y rendre en ballon libre lors de son mémorable voyage du 28 juillet 1890 , un mois jour pour 
jour après son mariage, en compagnie de son épouse Aline de Launay et de son cousin Gabriel Gaupillat ; il ont en effet atterri - en catastrophe et non sans mal - à Sains, dans l'Aisne, tout près de la frontière (MARTEL 1891).

Le premier contact avec un correspondant Belge dont nous ayons trouvé trace, parmi les Archives Martel, date du 16 janvier 1890. C'est un pionnier de la spéléologie de ce pays qui s'adressait à Martel: Alphonse Collignon. L'on sait qu'il explora chez lui beaucoup de cavités, et notamment celle qu'il chérissait le plus, la grotte de Rochefort, qui, grâce à lui, fut l'une des premières cavernes aménagées d'Europe. Le fils du correspondant de Martel, Armand Collignon, fut l'un des 121 membres fondateurs de la Société de Spéléologie.

Voici la transcription de cette lettre historique, qui fixe le tout début des très longues relations épistolaires qui devaient s'instaurer entre Martel et les spéléologues Belges.

"Belgique Rochefort le 16 Janvier 1890

Monsieur Martel à Paris.

Je viens Monsieur vous remercier bien vivement de l'envoi que vous avez bien voulut m'adresser de la belle découverte d'une magnifique Grotte (la grotte de Dargilan) qui promet un bel avenir et qui ne pourra que gagner pour les nouvelles découvertes que vous ne cesserez de faire, votre nom y sera comme une Gloire éternelle.

Je vous remercie de vous être rappelé à une visite faite aux Grottes volcaniques de Rochefort que j'ai découvertes et que sans mes 81 ans je pourrais plus que doubler de curiosités \& d'un lac navigable de plus de six cents mètres, des salles d'une très grande dimension me sont connues et d'une hauteur prodigieuse avec des pétrifications jusqu'aujourd'hui encore inconnues pour la formation de formes si extraordinaires qu'on ne peut se figurer que la Nature ait fait des Merveilles pareilles.

L'une (sic) trois longues ailes minces de formes irrégulières descendent, donnant chacune un son différent en les réunissant finit par donner un accord parfait.

Une autre salle passant en dessous d'un dôme parfaitement formé dont le dessus est garnit de bien belles décorations de pétrification sayant des formes aussi de poissons pétrifiés.

Mes explorations m'ont arrêté devant une salle d'une très grande dimension dont l'air éteignait mes lumières et impossible de s'y introduire qu'en arrairant (lire bien sûr " aérant") cette immense curiosité, j'ai même faiblit par la force du gaze dont je voulais me rendre maître.

Ont pourrait donc établir une navigation par barque de plus de six cents mètres si mon grand âgen'y mettait obstacle.

Ont pourrait lui donner un développement à y passer des journées.

Je vous remercie encore de votre attention et vous présente Monsieur, l'assurance de ma parfaite considération.

Cette lettre a été très difficile à lire, parce que l'écriture en est presque effacée. Le style décousu et les fautes d'orthographe de son auteur découlent probablement de son grand âge, mais aussi d'une instruction rudimentaire qui, de plus, ne lui permit pas de comprendre que la caverne n'était pas d'origine ignée.

A la suite de cette lettre, Martel et Louis de Launay visitèrent en détail ces deux cavernes le 3 avril1890. Martel était venu dans ce pays, à la demande de son ami l'avocat Crépin, pour donner une conférence au Club Alpin Belge, à Bruxelles. Martel 
et son futur beau-frère en profitèrent pour descendre des rivières au fil de l'eau (leur embarcation en toile, de fameuse marque " osgood ", s'éventra à deux reprises ce qui la rendit inutilisable).

Apparemment, Martel ne devait retourner en Belgique que huit ans plus tard, toujours pour y donner une conférence (à la Société de Géographie de Belgique, le 23 avril 1898) ; le sujet portait sur ses explorations spéléologiques en France,Autriche et Irlande. Le lendemain, il en profitait pour établir le plande la grotte de Rémouchamps (où il fit un peu de navigation), avec Ernest Van-den-Broeck qui était, semble-t-il, pour la première fois associé à l'une de ses investigations. Van-denBroeck (1851-1932) était, depuis plus de dix ans en 1898, Secrétaire Général de la Société belge de Géologie, de Paléontologie et d'Hydrologie, dont il avait été l'un des principaux fondateurs en 1887. Entre autres distinctions et fonctions, il fut Conservateur du Musée Royal d'Histoire Naturelle de Belgique. Il était donc une importante personnalité scientifique.

Le choix de cette grotte comme première caverne étudiée par Martel n'était pas dû au hasard, puisque, bien plus tard, dans une lettre du 5 novembre 1906, Van-denBroeck déclarait :" La grotte de Rémouchamps et surtout la vallée des Chantoirs constituent, comme vous le savez, l'un des clous de notre livre au point de vue surtout de l'hydrologie de nos calcaires dévoniens ".

Le lendemain 25 avril 1898, il donna sa troisième conférence en Belgique, à la Société de Géologie de Bruxelles, sur 'L'exploration scientifique des cavernes, de la spéléologie et des résultats de dix années de recherches dans les grottes,les abîmes, etc., de l'Europe", ceci, bien entendu, à l'instigation de Van-den-Broeck.

En compagnie de celui-ci, il continua l'étude de la grotte de Rémouchamps au mois de mai suivant, ce qui semblerait indiquer qu'il avait fait un séjour en Belgique d'au moins une dizaine de jours.

De cette expédition souterraine faite en commun, en deux séances donc, Van-denBroeck tirera un article, cette même année 1898: Sur la rivière et la grotte de Rémouchamps : note préliminaire sur ses niveaux à silex et à ossements d'âge paléolithique dans le Bulletin de la Société d'Anthropologie de Bruxelles (1er juin 1898,16 p.).

Manifestement intéressé parles phénomènes karstiques de la Belgique, Martel y revint en septembre de cette même année. Cette fois-ci, il avait jeté son dévolu sur le système karstique de Han-sur-Lesse, objet de son voyage " initiatique " de 1888. Le 18 septembre, à 16 heures, toujours avec Ernest Van-den-Broeck, il fit une coloration au célèbre Trou de Belvaux, avec $1 \mathrm{~kg}$ de fluorescéine.

Le lendemain, avec son collaborateur attitré pour la Belgique, il se rendit à nouveau à la grotte de Han-sur-Lesse; à midi, ils virent arriver la caractéristique coloration verte au pont du Styx (salle de la Place d'Armes) ; à 16 heures, ils observèrent le passage de la fluorescéine à la sortie de la caverne, soit 24 heures après la coloration au Trou de Belvaux.

Les recherches hydrogéologiques belges, dont Ernest Van-den-Broeck allait devenir le grand spécialiste, débutaient donc par ce coup d'éclat, recherché médiatiquement par Martel.

Le 20 septembre suivant, avec son collaborateur, il découvrit dans cette caverne deux siphons dont un, trouvé désamorcé, fut franchi à la nage $(0,20 \mathrm{~m}$ de hauteur et $0,60 \mathrm{~m}$ de largeur) ce qui permit d'ajouter dix mètres de galeries seulement, mais qui 
fut présenté comme un exploit, médiatisé comme il se devait.

Le 25 octobre 1898, la Belgique attira une nouvelle fois Martel qui, à 20h30, donna une conférence (sa quatrième dans ce pays) à la Société belge de Géologie,de Paléontologie et d'Hydrologie de Bruxelles, conjointement avec Ernest Van-denBroeck,sur les "Nouvelles recherches et constatations à Han-sur-Lesse ".

Visiblement, Martel cherchait à se faire un nom dans ce pays voisin de la France, qui est l'un des plus caverneux d'Europe.

L'on sait que l'année 1900 vit revenir Martel en Belgique; il s'y illustra parla découverte de la grotte sépulcrale dite "Trou-du-Crâne ", laquelle est située à proximité du Trou-du-Frontal.

L'année d'après, le 10 octobre 1901, il examina seulement l'entrée du Trou-quiFume.

Quelques mois plus tard, il décidait de s'intéresser vraiment à la Belgique, probablement dans le dessein d'emmagasiner des données en vue d'un gros ouvrage dont il avait conçu le plan, et pour lequel il s'était assuré du concours des deux plus actifs spéléologues de Belgique du moment: Rahir et Van-den-Broeck (ceux-ci s'étaient connus et appréciés lors de travaux en commun au site préhistorique de Furfooz). Le prétexte à sa venue dans le royaume était une excursion spéléologique organisée conjointement par la Société de Spéléologie et par la Société de Géologie de Belgique, laquelle, sur proposition de Van-den-Broeck, devint le relais, dans ce pays, de la première citée.

Le 20 mai 1902, il se rendit donc à Bruxelles où il commença par faire une conférence, sous les auspices de la Société Belge de Géologie, sur ses recherches dans les Alpes Dauphinoises et sur les plateaux de Vaucluse, de la Drôme, de la Lozère et du Lot. Il en profita pour étudier les phénomènes du calcaire de la Belgique, jusqu'au 31 mai. C'est ainsi que le 23 mai, il établit la topographie de l'abîme de Comblainau-Pont et de la grotte de Tillf ; que le lendemain, il fit l'exploration de l'Abîme de la Carrière de la Dentine (- $20 \mathrm{~m})$, ainsi que celle du Trou du Diable, à Ramioul, avec Collard, Rahir et Van-den-Broeck; le 25 mai, il prit des photographies au fond du Trou-Manto, visité en compagnie de Jeand'Ardennes (pseudonyme d'un journaliste), Jules de Baugnies, Dommartin, Victor Martin, Rahir et Van-den-Broeck ; le lendemain, il établit la topographie de la grotte du Pré-au-Tonneau, près de Rochefort, et le sur lendemain, celle de la grotte d'Eprave ; le 30 mai fut consacré à des relevés de température dans la Lesse,l'Aiguigeois des Sources et le Trou-de-la-Loutre, et le 31 mai, il établit les topographies de la grotte-résurgence de l'Eau Noire, à Nismes et du Trou de l'Abîme à Couvin. En outre, l'on sait qu'à cette occasion, il prit des photographies dans la Salle des Mamelons de la grotte de Han-sur-Lesseet dans la grotte de Remouchamps; il effectua aussi les topographies du Trou des Nutons à Furfooz, du Trou Méria, près Celles, du Trou des Veaux et de la caverne ossifère d'Engihoul, près d'Engis.

On ne sait s'il est revenu quelques jours en France, où s'il a fait du tourisme nonspéléologique jusqu'aux 16 et 17 juin 1902, journées où il s'occupa d'une coloration au Trou-de-la-Loutre avec Edmond Rahir et Verhas.

Préoccupé par la rédaction de chapitres du livre commun sur la Belgique, Martel revint dans ce royaume le 21 décembre 1902 pour des relevés de température au Trou-qui-Fume. Dix jours plus tard, il réitérait ses mesures dans cette même cavité et en faisait d'autres au Puitsd es Vaux. 
En outre, l'on sait que cette mémorable année “ belge " de 1902 fut l'occasion, pour lui, de la découverte du Trou de la Mâchoire, sise à côté du Trou-du-Frontal (bassin de la Lesse).

En 1903, il était présent au Congrès de Bruxelles de l'Association Française pour l'Avancement des Sciences. Le 18 janvier de cette année-là, il fit des relevés de température au Puits des Vaux et au Trou-de-la-Loutre ; le 28 mai 1903, il établit la topographie de la grotte du Trou-Maulin, près de Rochefort.

Il y a lieu de signaler ici l'épais fascicule auquel il collabora activement et qui fut publié en 1904 à l'instigation de chercheurs belges, et qui a été rapidement épuisé, avant le mois de juin 1905 : L'étude des eaux courantes souterraines :-eaux alimentaires en région calcaire - Par l'emploi des matières colorantes-fluorescéine Bull. Société Belge de Géologie, tome XII, Bruxelles, Hayez, 1904, 218 p.(tirage à 200 exemplaires seulement). Il ne semble pas, par ailleurs, qu'il ait mis les pieds en Belgique en 1904.

Par contre, l'année d'après, on trouve sa trace, le 20 juillet 1905, à la Fontaine Patenier où il fit des mesures de températures. Avant le 19 octobre 1905 (jour où il donna une conférence à la Société Royale Belge de Géographie, sur "Les cavernes, les abîmes et les rivières souterraines de la Belgique ") peut-être en présence du prince Albert de Belgique - comme il était prévu—, il explora, en compagnie de Van-den-Broeck, Rahir et Loé, le Trou de la Machoire, dans le site de Furfooz ; il y découvrit une sépulture préhistorique (plus quatre autres dans des cavités voisines). Le 20 octobre, avec les mêmes, ce fut au tour de la grotte de Montfort, près de Furfooz, puis de la grotte de Bouvignes, ensuite, le lendemain, de la grotte des " fonds de Leffe" ainsi que la grotte de Dinant. Peu après,outre l'exploration de la perte du Trou de l'Abîme, il s'intéressa aux célèbres abîmes fossiles des Abannets de Nismes,près de Couvins, à l'invitation personnelle de Van-den-Broeck : “ je pense que l'on pourrait aussi vous signaler la région dite des Abannets, de Couvin! Vous n'avez pas vu les plus charactéristiques et le plus profond de ces immenses abîmes, sièges naguère d'exploitation de mines de fer et que je suis amené à considérer comme l'équivalent des poches à phosphates du Quercy ".

Le 23 octobre, il donna la même conférence à la Société d'Etudes coloniales d'Ostende, à $20 \mathrm{~h} 30$.

Il avait projeté de revenirle 15 mai 1907, mais, à cause d'une tracasserie venue de Van-den-Broeck, il se récusa le 11 courant, vexé.

Il ne revint en Belgique que le 2 février 1908 pour des relevés de température à la fontaine de Saint-Hadelin et à la Fontaine de Mahenne. On ne sait si son séjour était long, entre février et mars 1908 ; toujours est-il que le 26 février il effectuait des mesures de température à la Fontaine Patenier, le 9 mars il étudiait la fontaine de Saint-Hadelin, ce jour-là en extraordinaire crue, le 10 la fontaine de Saint-Hadelin (en crue exceptionnelle également), et, le 12 mars 1908, la Fontaine de la Baronne.

Il faut noter, tout de même, qu'il ne s'est pas déplacé en Belgique à l'occasion de la sortie du livre sur “Les Cavernes... de la Belgique ", probablement en raison des relations extrêmement tendues entre ses deux collaborateurs.

Il ne devait apparemment revenir en Belgique que bien plus tard, en 1922, pour participer au XIII ${ }^{\circ}$ Congrès Géologique International de Bruxelles. 


\section{LA LONGUE GESTATION DE L'OUVRAGE, SUR FOND DE POLÉMIQUE CHRONIQUE}

Cet ouvrage fondamental pour la spéléologie européenne est issu d'un accouchement infiniment difficile... L'étude de la correspondance est, sur ce point, fort révélatrice du climat délétère dans lequel le livre "Les Cavernes et rivières souterraines de la Belgique " a été conçu et mené à bien. Même si la part qu'y a prise Martel est minoritaire, il n'est pas douteux que, de tous les écrits faits en collaboration avec d'autres auteurs, celui-ci fut le plus pénible dont il ait eu à s'occuper.

Aux complications inhérentes à l'éloignement des coauteurs, à l'obligation de collaborer par correspondance, aux incessantes modifications, relectures,compléments, retraits, corrections, s'ajoutèrent des difficultés d'ordre relationnel qui empoisonnèrent, n'en doutons pas,l'existence de Martel au moins de 1905 à 1909 ; ceci, justement dans une période de troubles chez lui, tant financiers que psychologiques.

Pour l'édification,tant des spéléologues belges que des historiens de la spéléologie, il n'est pas superflu de se plonger dans cette vaste correspondance, piochant de ci de là des informations variées, afin d'y voir plus clair.

Le premier échange épistolaire avec Ernest Van-den-Broeck date du 26 février 1898, en réponse à une lettre de Martel du 7 février (non retrouvée). On ne sait pas si les deux hommes s'étaient déjà rencontrés auparavant et s'ils avaient eu l'opportunité d'excursionner ensemble. Van-den-Broeckintroduit sa lettre d'un "Mon cher Monsieur Martel", formule qui cédera le pas à "Mon cher Ami" dès le 22 septembre 1898, après la venue de Martel en Belgique pour étudier la grotte de Rochefort.

Ce probable premier contact entre les deux hommes était motivé par le projet de deux conférences de Martel à la Société de Géographie et à la Société de Géologie de Belgique, conférences qui eurent lieu effectivement, devant 500 et 300 personnes. Comme on la vu plus haut, Martel en profita pour aller inspecter la grotte de Rémouchamps.

Outre l'envoi de documentation spéléologique (tirés à part, fascicules de Spelunca) pour parfaire ses connaissances, Van-den-Broeck demandait à Martel, dans cette lettre de premiers contacts, de rédiger une étude spéciale sur les cavernes belges, étant donné son expérience;il lui faisait part aussi, en géologue, de ses divergences de vues à propos d'actions mécanique et corrosive dans la karstogenèse, ainsi que, et surtout, sur la réalité de l'existence, dans certains calcaires du Carbonifère, de vraies nappes d'eau.

Suite à la réception des mémoires de la Société de Spéléologie alors parus, Vanden-Broeck résolut, dans la perspective de le publier dans cette série, de se mettre à l'établissement d'un inventaire des grottes belges, dont il estimait le nombre à 5 ou 600. C'est ce travail-là qui a servi de base au livre de 1910.

Les premiers vrais pourparlers pour l'élaboration du livre eurent lieu fin mai 1902, à la fin de l'active campagne en Belgique auquel Martel fut associé. La toute première lettre qui en fait état date du 10 juin 1902 ; elle est signée de Van-den-Broeck : “M. Rahir s'offre à nous préparer le gros œuvre et à nous éviter du travail matériel. Il nous préparera la besogne".

Une carte postale de Rahir, (26février 1903), montre qu'à cette date les deux correspondants attitrés de Martel pour la Belgique n'avaient pas encore débuté leurs rédactions pour le grand ouvrage : "Monsieur Van den Broeckavait justement l'in- 
tention de vous écrire pour vous demander vos notes spéléologiques sur l'expédition de l'année dernière en Belgique. Nous sommes disposés, et moi tout particulièrement,à nous occuper de la rédaction du volume sur les Cavernes et les rivières souterraines de Belgique. Aussitôt que nous aurons reçu vos notes nous y travaillerons ". L'année d'après, le 19février 1904, Van-den-Broeck indiquait : " notre livre sur la Belgique avance ferme! Rahir se donne beaucoup de peine. Ma collaboration viendra plus tard, mais déjà je lui ai passé l'ensemble de mes dossiers personnels ”.

Rahir était, à cette heure, le responsable centralisateur des écrits destinés au volume en gestation. En mars 1904, il estimait la fin des rédactions proche et pensait que l'ouvrage, de format" in-octavo ", atteindrait 400 pages... Martel avait été chargé de trouver un éditeur à Paris ; il s'était adressé en vain à Charles Delagrave. Edmond Rahir, de son côté, avait sollicité la Maison Lebègue, en Belgique, qui s'était récusée. Il en fut de même pour les maisons Dunod, Gauthier-Villars, Masson,etc.

Le 17 février 1905,Van-den-Broeck, entré en possession du manuscrit de Rahir, disait à Martel : “ le manuscrit vous parviendra sous peu puis j'aurai à m'en occuper à mon tour pour compléter ce que Rahir y a déjà introduit d'après mes notes et documents ".

Etant donné les difficultés que rencontrèrent les trois spéléologues pour trouver un éditeur, ils songèrent à une parution à compte d'auteurs, avec subventions de mécènes et souscriptions d'administrations belges. Van-den-Broeck, grâce à ses nombreuses relations de marque en convaincra plusieurs ; au final, il apportera lui même 2000 $\mathrm{F}$ et Rahir environ $1200 \mathrm{~F}$;Martel, en difficultés financières à cette époque, ne remettra rien. Il déclarera simplement ne vouloir percevoir aucun droit d'auteur pour la première édition.

Rahir voulait tirer à 1000 exemplaires, tandis que son concitoyen envisageait 1200 ou 1500 , pensant qu'une réédition ne pourrait se présenter de sitôt.

Afin de pouvoir obtenir des fonds de la part de mécènes et de divers ministères, Van-den-Broeck envisagea un scénario audacieux : " tenter d'obtenir le patronage de S. A. le Roi. Peut-être cela nous incitera-t-il à tirer à 1500 ” (lettre du 12 mars 1905). Acette fin, il provoqua une conférence de Martel à la Société Royale Belge de Géographie : “ c'est surtout la haute protection royale que votre conférence - bien entendue et bien dirigée - pourrait nous faire obtenir. Jusqu'ici, S. M. Léopold a demandé du temps avant de prendre sa décision au sujet de la dédicace du livre et je tiens à vous expliquer comment il se fait que vous tenez entre vos mains, par cette conférence, le reste de la décision royale! La dédicace obtenue c'est le succès assuré! "

Pour sensibiliser le souverain belge, Van-den-Broeck ne manquait pas d'imagination: " l'idée d'un exemplaire relié pour le Roi me paraît excellente. Je suis en excellents termes avec un avocat-député (de la majorité) qui est le frère de son Secrétaire particulier et que je vais charger des négociations. Le Roi s'intéresse beaucoup et depuis longtemps aux questions d'eaux alimentaires ”. (...) “J'attends donc vos documents pour l'Exposition et l'Avant-Propos dans lequel vous avez sans doute tenu compte de ce que vous a demandé Rahir " (lettre du 14 mars 1905).

Par la suite, après une entrevue que Van-den-Broeck eut avec le Secrétaire particulier de Sa Majesté Léopold II (M. Carton de Wiart), la perspective d'une dédicace du livre au Roi semblait retenue. Il est à noter que le livre devait bien obtenir cette très haute protection, non du Roi Léopold II (mort en 1909), mais de son neveu, le Roi 
Albert 1er, comme l'ont attesté plusieurs mentions journalistiques en Belgique à la parution.Van-den-broeck disait, d'ailleurs: “ je vous adresse aussi la copie de la lettre que m'a fait adresser $S$. A. R. le Prince Albert, qui accepte de parrainer notre œuvre" (lettre du 5 avril 1909) ; mais encore : " il est vrai que notre haut protecteur le Roi Albert a bien voulu faire savoir à nos divers Ministres qu'il lui serait agréable que bon accueil soit fait à mes requêtes" (lettre du 7 juillet 1910).

Puisqu'on s'acheminait vers un ouvrage marquant, intéressant un pays entier, susceptible d'attirer l'attention d'éminentes personnalités, des rivalités ne pouvaient qu'apparaître entre les trois coauteurs soucieux individuellement de leur notoriété et de leurs carrières respectives.

Un soupçon de polémique apparaît clairement dans une lettre de Rahir, datée du 17 mai 1905: “ s'il y a un retard dans la publication de notre livre, ce n'est ni à cause de vous ni à cause de moi, mais bien de notre excellent collaborateur V. D. B. qui souvent entreprend beaucoup trop de travaux à la fois. Sans cela, la publication aurait pu commencer depuis deux mois ".

Le 31 mai, Rahir reçut l'Avant-Propos de Martel, ce qui était le signe d'une proche sortie en librairie...

La parution soi-disant imminente de l'ouvrage "Les cavernes de la Belgique" avait même été annoncée dès le 1er juillet 1905, dans la Revue la Nature : “parầtra l'automne prochain, chez Dunod, à Paris ”. Pourtant, Van-den-Broeck, le 11 septembre1905, demandait à reporter la parution de l'ouvrage au printemps 1906, parce qu'il avait jugé bon de reprendre la totalité des textes, lesquels, à ses yeux de géologues, n'étaient pas assez scientifiques...

Une lettre de Rahir (13 septembre 1905), au contenu très réaliste, prophétise une apparition très tardive de l'ouvrage: “ je ne sais pas quand notre livre paraîtra? Cela m'attriste fort parce que j'avais travaillé d'arrache-pied pour le faire paraître en Octobre. Pas plus que moi vous n'êtes responsable du retard très, même trop considérable que va subir cette publication par suite de bouleversements, nombreuses et importantes modifications après coup (etc.) que lui apporte notre ami V. D. B. Et dire que le manuscrit est entré en sa possession pendant des mois. Tout cela est décourageant. V. D. B. ne sait pas se limiter et je ne m'étonnerais pas si le livre ne paraissait que dans un an. Si vous avez quelque influence sur lui, vous me feriez plaisir en lui conseillant de s'efforcer de limiter son débit ainsi que la recherche de trop nombreux faits nouveaux qui ne sont que la répétition des précédents ".

Van-den-Broeck, d'ailleurs était lui-même pessimiste sur la sortie de l'ouvrage prochainement : " d'une part,disait-il, je constate les extrêmes lenteurs de l'imprimerie, d'autrepart, je me rends compte de ce qu'il me reste à faire, surtout pour la partie purement hydrogéologique et d'eaux alimentaires".

Ayant fait part de ses soucis à Rahir, celui-ci lui déclara devoir les partager et accepta même "l'impérieuse nécessité de bouleverser la suite du texte" mais il demanda " à être déchargé de tout travail supplémentaire ", sauf l'examen des épreuves. Van-den-Broeck avouait: "certes, je reconnais qu'il a largement, l'ami Rahir, fourni sa part de collaboration" (lettre du 14/09/1905).

Ce refus de la part de Rahir était stratégique : il voulait obliger Van-den-Broeck, privé de son concours, d'abdiquer et de donner aux imprimeurs les textes vus et revus, tels quels,sans adjonctions pléthoriques journalières. Et surtout, Rahir ne voulait pas payer de ses deniers aux imprimeurs le produit du travail intarissable de son 
compatriote.

Même s'il admit ce dernier point, Van-den-Broeck prit ombrage de cette attitude. Sa rancœur envers Rahir allait enfler de jour en jour, jusqu'à la rupture définitive et l'enlisement du " projet".

Un exemple de ce qu'étaient leurs relations " amicales" : "vous paraissez oublier, mon cher Monsieur Van den Broeck, que bien souvent, lorsque j'ai eu des explications verbales avec vous, j'ai dû subir vos gros mots et vos colères injustifiées et que, de plus, vous teniez alors constamment le crachoir, m'empêchant absolument de placer en mot ", "Cordiale poignée de main de celui que vous qualifiez, même sur carte postale, de déséquilibré ! de Grand Lama, devant lequel tous doivent se plier! d'irresponsable, etc. etc. ".

Quant à Martel, il préparait en secret dès avant 1905, pour le faire paraître fin1907, l'énorme ouvrage bibliographique “La Spéléologie au $X X^{\circ}$ siècle ". C'est dire combien le surcroît de travail que lui imposait Van-den-Broeck ne l'enchantait guère et qu'il traînait les pieds... Celui-ci, par la force des choses, pensait ne pas pouvoir faire sortir le livre avant octobre 1906 : nouveau report, donc.

Ernest Van-den-Broeck cherchait-il à avoir carte blanche pour avancer tout seul, à sa guise, vers l'idéal qu'il s'était fixé ? Toujours est-il qu'une lettre qu'il reçut de Rahir lui donna les coudées franches, même si son amour-propre en fut blessé : “ je serais désireux de n'avoir plus sur les bras la question financière. Vous pouvez me refuser, c'est votre droit. Si cela devait vous décider à accepter ma proposition, je serais disposé à faire abandon à votre profit de tout bénéfice éventuel sur la vente du livre. De cette façon vous ajouterez toutes les planches que vous désirez, vous allongerez le texte tant que vous voudrez et le livre paraîtra quand il vous plaira". Rahir fut obligé de payer, mais ne fut plus tenu à écrire le moindre texte. Son concours iconographique était seul requis.

Fin décembre 1905, début janvier 1906, Van-den-Broeck, pris à la gorge, démissionna de cinq sociétés savantes et abandonna la charge de secrétaire général de la Société Géologique de Belgique qu'il occupait depuis vingt années : il allait, disaitil, ne s'occuper plus que du livre. Appelé à faire un arbitrage entre Rahir et Van-denBroeck, Martel leur adressa un ultimatum : si tout se passe bien, il achètera pour 100 ouvrages, affirmait-il,pour son usage personnel ; il ne voulait plus recevoir de lettres ressassant leurs acrimonies respectives ; il les menaçait de cesser toute relation amicale et de reprendre à son compte personnel, pour non exécution des conventions, les textes qu'il avait remis quatre années auparavant.

Ernest Van-den-Broeck travailla effectivement d'arrache-pied, toute cette année 1906, non sans offenser au passage Rahir en remaniant, presque sans exception, tous ses textes, et non sans empiler sans arrêt des feuilles nouvelles ! Plus tard (lettre du 24/12/1907), il devait même avouer, non sans malice, qu'il n'avait laissé intact qu'un texte de Rahir sur le Vallon de Lesves, ceci parce que Martel commençait à protester!

En fait, il reprenait scrupuleusement la plupart des écrits de Rahir pour asseoir sa prééminence au sein du trio d'auteurs. Dans chaque lettre qu'il écrivait à Martel, il redoublait de petites ou longues phrases assassines, surtout en septembre 1906 où,cérébralement fatigué, dépressif, il fâcha son concitoyen qui ne lui donnait plus que du "Monsieur", et non plus du " cher Ami " comme auparavant! A ce train-là, Rahir ne voulait plus rien corriger, et on peut le comprendre ! 
Van-den-Broeck était très explicite là-dessus: “ quand j'ai bénévolement accepté de laisser mon nom tout à la fin c'est quand, par excès de conscience, je me demandais si j'aurais jamais le temps de m'occuper sérieusement du livre que nous avions tous deux (vous et moi) décidé de faire ensemble et pour lequel Rahir nous a ensuite offert sa collaboration. Mais tout est bien changé !! ! J'ai transformé non seulement le plan général de l'ouvrage(ordre géologique au lieu de géographique), mais toutes ses parties passées par le crible d'une étude géologique approfondi, ce qui était de mon domaine spécial. Certes j'ai beaucoup " allongé ” mais au lieu du livre simplement pittoresque et intéressant du début, j'ai fait, avec votre collaboration, un traité peut-être moins attrayant, mais qui restera absolument utile et formera un petit monument où l'on pourra puiser largement".

Van-den-Broeck ne voyait en Rahir qu'un bon cartographe et dessinateur et, à la limite,qu'un bon descripteur des sites naturels. Aussi tenta-t-il depersuader Martel qu'il convainque Rahir d'accepter les modifications radicales faites à ses textes, mais que, néanmoins, il fournisse des figures supplémentaires !

Après cela, il affirmait que la partie allait être gagnée et que le livre serait en passe d'être publié...

Rahir, se ravisant, ne voulait probablement pas être écarté définitivement de l'ouvrage : il accepta donc de travailler avec Van-den-Broeck, à condition que celui-ci se déplace chez lui pour l'œuvre commune.

Rahir et Martel étaient impatients que le livre paraisse enfin ! Ils allaient pourtant devoir encore patienter presque quatre années de plus !

Martel venait de terminer son gros fascicule "L'eau, Etude hydrogéologique " et préparait son ouvrage "L'Evolution Souterraine ", qui devait paraître en1908, sans parler de son énorme "Spéléologie au $\boldsymbol{X} \boldsymbol{X}^{\circ}$ siècle" dont les corrections devaient lui prendre beaucoup de loisir, et sans oublier, enfin, ses deux épais fascicules totalisant 200 pages sur les "Pyrénées Souterraines ", soit en tout plus de 1400 pages, rédigées sans l'aide de quiconque. Il en avait plus qu'assez des jérémiades de son correspondant.

Voici quelques échantillons des amabilités distillées par Van-den-Broeck : “ $D e$ grâce, faites en sorte de mâter l'hostilité et le mauvais esprit de Rahir qui est déjà responsable de toute cette dernière phase de retard et d'arrêt suscitée par ses inconcevables revendications. Je suis à même d'ailleurs demarcher sans lui et sans son concours! Depuis de très longs mois d'ailleurs il n'a absolument plus rien fait pour m'aider. Tout ce que je demande c'est que grâce à vos bons avis il ne me mette plus de bâton dans les jambes... sans quoi je désespérerai d'arriver au port. "

Il serait inutile de détailler les péripéties survenues de 1909 à 1910, année effective de la parution, non d'un volume, mais de deux, tome 1 et tome 2 ; on retrouve, dans la correspondance, les mêmes leitmotivs ressassés mois après mois, semaine après semaine, de 1905 à 1908.

On retrouve un Van-den-Broeck toujours aussi prolixe, toujours aussi près du but, toujours aussi pris par ses mille occupations étrangères au "livre" ; Rahir est bien sûr son bouc émissaire favori, mais il s'en prend aussi à G.Cosyns, le père de Max, celui-ci étant bien connu pour sa participation à l'épopée de la Pierre-Saint-Martin, dans les Pyrénées basques. Il faut noter une toute petite accalmie entre Rahir et Vanden-Broeck, en septembre 1908, provoquée par Martel qui avait résolu de ne plus répondre aux lettres de ce dernier, estimant qu'il se moquait de ses collaborateurs en 
ne tenant pas ses engagements de faire paraître au plus tôt : Van-den-Broeck, ayant eu peur d'être totalement lâché, avait persuadé Rahir de revenir à de" meilleures intentions "... pour peu de temps, car on a même frôlé la catastrophe en décembre, peut-être même un duel entre les deux belges, qui sait ! En effet, voici ce que devait déclarer Van-den-Broeck: “ Pratiquement les moyens violents que préconise Rahirn'aboutiraient au contraire qu'à un véritable esclandre et si celui-ci devait, comme c'est fatal, rejaillir sur mon avenir tout entier, je n'oserai garantir de garder mon sang froid et... hélas, je me connais,peut-être qu'un irréparable malheur, dû à la vengeance, en serait la conséquence! Tout en me faisant un devoir de dominer la violence de mes sentiments, je sais qu'en pareille circonstance (car cela s'est déjà présenté pour une autre affaire), je vois rouge facilement et bien malgré moi!! ".

Quant à Martel, tiraillé entre Van-den-Broeck et Rahir, lequel lui avait sympathiquement demandé d'écrire la préface d'un nouveau livre de son cru (ce que l'intéressé avait d'ailleurs accepté, mais qui déplaisait souverainement au troisième acolyte), il bouillait d'impatience... tant et si bien qu'il craqua, un jour (8 mars 1909), et dit son fait au vibrionique géologue bruxellois dans un de ses courts avertissements sans appel dont il avait le secret: "le ton de votre lettre est inacceptable. Oui, je ferai la préface à Rahir. Il ne tient qu'à vous de faire paraître le livre avant. Voilà trop longtemps que vous abusez de notre patience.C'est fini ! Elle est àbout!!'”. Hélas, comme Rahir avait projeté de faire paraitre son ouvrage en octobre et qu'il était pressé, Martel ne put lui remettre la préface promise.

Vint enfin le jour tant attendu par Ernest Van-den-Broeck de l'achèvement de l'ouvrage : " la date du dimanche 2 mai 1909 est une date mémorable dans mon existence de ces dernières années, car c'est celle m'ayant permis d'écrire... avec quel soupir de satisfaction... le bienheureux mot: fin sous la dernière ligne rédigée de notre chapitre final XVIII!".

Van-den-Broeck s'était démené comme un diable pour trouver un financement; il avait notamment su attirer 220 souscripteurs.

Le tome 1 des " Cavernes... de la Belgique" parut fin novembre 1909 (un mois après l'ouvrage non préfacé de Rahir, donc), et le tome 2 vers le 15 décembre suivant.

\section{MAIS QUE S'ÉTAIT-IL DONC PASSÉ ?}

Il s'était passé ceci : Van-den-Broeck souhaitait se faire remarquer, pour sa carrière. Dans un premier temps, il ne s'était pas aperçu que la réalisation de cet ouvrage, de portée nationale, était susceptible de le mettre en valeur. Il s'était donc contenté de documenter Rahir qu'il avait chargé d'écrire la plupart des chapitres et de réunir l'iconographie.Martel dirigeait officiellement l'ouvrage, fournissait quelques chapitres et des portions de textes sous formes de gloses plaquées, de ci, de là, sur les écrits qui lui seraient soumis pour avis et corrections.

Dans une lettre, on relève que Van-den-Broeck avait envisagé (en février 1905) de répartir les bénéfices éventuels de l'ouvrage ainsi : $50 \%$ à Rahir, $25 \%$ à Martel et $25 \%$ à lui-même. Il semblerait donc qu'à lui seul Rahir avait, avant 1905, mis en forme à lui tout seul la moitié de l'ouvrage.

A l'origine, il avait été prévu de signer l'ouvrage dans l'ordre suivant : Martel, Rahir et Van-den-Broeck, soit selon l'ordre alphabétique : “j'ai fait placer mon nom 
en dernier lieu en utilisant pour cela l'ordre alphabétique. C'est un hommage à Rahir que j'ai tenu à lui rendre, à cause de la plus grande peine qu'il s'est donnée pour élaborer le livre " disait franchement Van-den-Broeck dans une lettre à Martel datée du 2 juillet 1905.

De plus, il avait été convenu ce qui suit, comme l'a rapporté Rahir (lettre du 17 février 1908) : "dans l'Avant Propos que vous désirez, et avec raison, faire, la part de chacun ne doit pas être mentionnée (...). Le travail constitue un tout fait par les trois auteurs ; chacun apportant ce qui lui a été possible d'apporter. Pour les photographies, les plans, dessins, etc., ils sont des auteurs, à part, bien entendu, ceux qui porteront la désignation d'un autre auteur ".

Tout cela convenait à Martel qui, étant le plus expérimenté, était considéré par ses collaborateurs comme le maître, et eux les disciples. La deuxième place conférée à Rahir était justifiée par son labeur (descriptif et iconographique). Mais, en juin 1906, il n'en sera plus question : Van-den-Broeck estimera " équitable de passer avant Rahir ”, et Martel insistera plus tard pour que Van-den-Broeck passe avant lui-même. Van-den-Broeck, qui désespérait d'arriver à ce but, avait même, une fois, osé dire que son nom légal étant" Broeck ", qui signifie " Marais ", il devait avoir la première place suivant l'ordre alphabétique !

Comme Martel avait souhaité s'appuyer sur la parution de cet ouvrage pour faire forte impression sur les jurés chargés de désigner le lauréat du Grand Prix des Sciences Physiques de l'Académie des Sciences (remis avec en sus une forte prime), Van-den-Broeck se dit qu'il pourrait lui aussi s'en servir pour se valoriser en Belgique : " si j'accepte votre offre délicate ce ne sera pas par amour-propre mais parce que peut-être pour ma carrière et ma situation au Musée cela pourrait me rendre grand service (...) Si le livre pouvait paraître avec mon nom en tête il est certain que cela me vaudra sans doute la prochaine place vacante " pour espérer devenir Correspondant de l'Académie (dans un premier temps), puis Directeur du Musée Royal d'Histoire Naturelle de Belgique (en vain).

Il décida donc de doubler Rahir, très habilement, en reprenant tous les textes de celui-ci, les réécrivant et les complétant par une prose intarissable, sous prétexte qu'ils n'étaient pas assez scientifiques ni géologiques. Il augmenta aussi considérablement la cartographie,normalement dévolue à Rahir, n'hésitant pas à prendre sur lui de faire redessiner par un professionnel maintes cartes...

Rahir " faisait le mort", ne répondait plus aux sollicitations de Van-den-Broeck que si Martel le lui demandait comme un service personnel. Martel était donc très souvent prié d'intervenir, ce qui ne devait pas échapper à l'intéressé qui n'accédait aux désirs de l'un et de l'autre qu'avec de fortes réticences. Il est vrai que Rahir travaillait,discrètement, à des ouvrages de librairies, ou bien à des notices pour grottes aménagées, pour son compte personnel, ce qui lui laissait peu de temps pour accéder aux desiderata de Van-den-Broeck.

Après avoir dégoûté Rahir et obtenu sa défection pour les corrections, profitant aussi de l'éloignement de Martel,Van-den-Broeck finit par traiter tout seul avec l'imprimeur belge qui avait été retenu, et à qui les textes étaient remis au fur et à mesure de leur achèvement et traités en " placards" pour corrections : "l'imprimeur ne connaît que mes bons à tirer, car depuis longtemps il ne connaît que ceux-ci et c'est moi qui paie! ”, disait-il. Il ne soumettait past ous ses ajouts à Martel, probablement pour ne pas l'effrayer. Comme l'ouvrage était imprimé peu à peu, au fur et à mesure que 
Van-den-Broeck avançait dans ses productions hebdomadaires, celui-ci ne s'aperçut quie tardivement que sa minuscule écriture générait de nombreuses pages imprimées, par rapport à celles de Rahir et de Martel. Les 400 pages initialement envisagées furent vite dépassées alors que l'épaisseur des documents manuscrits à livrer à l'imprimeur était encore assez grande...

L’ouvrage a, par ces faits, été augmenté des deux tiers ! Ceci par la seule volonté de Van-den-Broeck qui escomptait bien que les deux autres auteurs lui offriraient la première place, une fois rendus à l'évidence de la supériorité numérique de sa production à lui.

Même si, à plusieurs reprises, il déclara s'incliner devant pareille suprématie, Martel rechignait à cette prétention,parce qu'il avait en vue le Grand Prix de l'Académie des Sciences et qu'il préférait que lui-même fût à la première place.

Puis en décembre 1906, Martel indiqua clairement à Van-den-Broeck qu'il ne voulait plus pour lui-même la première place parmi les auteurs. Peut-être espérait-il, par là, calmer son correspondant et obtenir un peu de tranquillité.

Van-den-Broeck disait ceci, le 3 septembre 1907 : “Je ne partage pas votre inquiétude au sujet du livre. Du moment que nous dépasserons les 4 ou 500 pages, ce n'était plus un livre à lire. Cela devient, avec un millier de pages, un livre d'études et à consulter et aussi pour les non spécialistes un livre à acheter pour cette bonne raison que grâce à nos généreux Mécènes nous mettrons en route pour 10 ou $12 \mathrm{~F}$ (car il faudra le cartonner) un livre richement et curieusement illustré, valant en librairie au moins une vingtaine de francs!" Van-den-Broeck était loin du compte, avec ses" mille pages "... aux quelles devaient s'ajouter, au cours des ans, quelques 700 autres!

La recherche incessante de mécènes lui permettra pourtant de penser pouvoir faire imprimer cet indigeste pavé (8000 F récoltés à la date du 3 septembre 1907; l'ouvrage, en 1910 , aura finalement coûté $35000 \mathrm{~F}$ ! !).

Comme nous n'avons pas, en règle générale, les lettres que Martel a écrites aux uns et aux autres, il est bien difficile de savoir quelle fut sa stratégie pour éviter l'implosion de l'ouvrage en gestation.Ce qui est sûr, c'est que toutes les lettres de Rahir respirent la plus grande franchise et expriment toutes la plus grande cordialité ; celles de Van-den-Broeck trahissent un esprit vaniteux,polémique, faussement admiratif de Martel, calculateur qui sait manier les phrases pour escompter des retournements de situation. Van-den-Broeck avait compris que Martel était muselé par son désir d'être reconnu par l'Académie des Sciences, et il en profitait et abusait de façon machiavélique.

Mais Martel savait bien juger les gens. Il devait être ulcéré par le comportement de cet homme qui était par ailleurs extrêmement brillant. Il rongeait donc son frein. Souvent, quand elles étaient longues ou trop finement écrites, il déclarait à son auteur n'avoir pas lu les lettres de Van-den-Broeck, histoire de lui faire comprendre qu'il ne fallait plus le harceler de sa prose.

Van-den-Broeck avait une écriture très désagréable à lire, qui fatiguait Martel, lui qui était myope ! En outre, ses lettres étaient outrancièrement longues et surchargées d'informations aux quelles il ne pouvait répondre par manque de temps ; leur lecture le fatiguait. Maintes fois Martel en fit le reproche à son collaborateur. Mais celuici lui rétorquait habilement et avec raison qu'il en était de même en ce qui le concernait : " hélas ma terrible écriture va bien vous tourmenter pour la lecture de cette 
longue lettre. Quel dommage que le temps manque pour la faire recopier par Mad Van-den-Broeck, qui a une écriture superbe qui nous fait la nique à tous deux " ! Car il est vrai que Martel était lui aussi difficilement lisible, sauf aux habitués.

Van-den-Broeck se connaissait très bien, puisqu'il déclarait: " tout en reconnaissant le bien-fondé des défauts dont vous m'accusez avec raison-et vous n'êtes pas le seul- je me reconnais, moi, incorrigible! La prolixité est en effet, dans le domaine scientifique, ma plus grande ennemie et c'est même, jusqu'à un certain point, une tare pour mes publications. Tout cela je le sais : mais le mal est organique, irrémédiable".

Quand il voulait vraiment se faire comprendre, Van-den-Broeck avait recourt, en plus de son épouse, à son secrétaire du Musée Royaldes Sciences, lequel calligraphiait excellemment.

Le 2 décembre 1907 survint un événement qui changea radicalement la donne : Martel fut enfin déclaré officiellement Lauréat de l'Académie des Sciences (Grand Prix des Sciences Physiques), après avoir concouru sur le sujet suivant dont il était le meilleur spécialiste français: "les Abîmes et les Cavernes, étude générale des eaux souterraines, notamment au point de vue de l'hygiène”. Désormais, il n'avait plus à se contenir pour dire son fait à Van-den-Broeck, qu'il avait pourtant pressenti pour devenir le Président de la Société de Spéléologie, probablement pourqu'il se décrispe et qu'il se sente en haute estime auprès de lui: “Dois-je vous dire combien profonde a été ma stupéfaction de me voir candidat au Conseil de la Société de Spéléologie pour le poste si élevé de la Présidence?" ; poste qui, si je ne me trompe, ne lui a pas été attribué, en dépit de l'appui de Martel (Lettre 3 décembre 1907).

A la suite de deux lettres reçues de Rahir (décembre 1907), dans lesquelles celuici révèlait de curieuses manières d'agir de son concitoyen, Martel adressa à ce dernier une lettre au contenu très vif s'achevant par une promesse de procès s'il ne mettait pas un termeaux rédactions de l'ouvrage et s'il ne cessait pas d'y faire, sans l'avis ni le contrôle des deux autres coauteurs, de perpétuelles et trop longues adjonctions.

Il faut dire aussi que Van-den-Broeck avait trop hardiment et maladroitement demandé à Martel qu'il veuille bien réserver,à titre d'avance, une partie de la somme qu'il lui avai tété allouée avec son prix de l'Académie des Sciences, pour l'édition de l'ouvrage commun... Or, les finances de Martel n'étaient pas mirobolantes ; en décembre 1907, cela ne faisait que deux années et un mois, grâce à la Revue La Nature dont il était devenu l'un des directeurs,qu'il percevait des revenus, depuis son abandon de sa charge d'avoué en avril 1899.

Van-den-Broeck fut obligé de venir à résipiscence, non sans avoir au passage égratigné Rahir, surnommé en passant "Croquemitaine ", parce qu'il postulait, contre deux candidats de poids, pour être nommé Directeur du Musée Royal des Sciences Naturelles et parcequ'il voulait que le livre paraisse: “ il me faut absolument avoir comme atout dans mon jeu l'apparition du livre avant la fin de ce trimestre".

En fait, Rahir a joué de nombreuses années durant le rôle du bouc-émissaire, de l'exutoire à la mauvaise humeur de Van-den-Broeck qui s'était un peu trop inconsidérément lancé dans un trop écrasant travail.

Loin de lui avoir ôté toute inspiration, il est certain, pour moi tout au moins, que cette lutte d'influence a poussé Van-den-Broeck à se dépasser pour affirmer sa suprématie spéléologique en Belgique. 
On constate que, quand la fin de la rédaction approchait,Van-den-Broeck n'aspirait plus qu'à la paix.

Paix d'abord avec lui-même, et avec Rahir.

La correspondance s'arrête brutalement, qui nous prive de toute information sur d'éventuelles reprises de bonnes relations entre les deux spéléologues belges.

\section{ENSEIGNEMENT, POUR MARTEL, ET POUR NOUS DES CAVERNES DE BELGIQUE}

Il est édifiant de constater que le principal auteur du livre" Les Cavernes de la Belgique ", Ernest Van-den-Broeck, avait lui même perçu que, par ses longueurs, ses répétitions, ses redondances, son œuvre serait très pénible à" digérer " par ses lecteurs qui, lassés dès les premières lectures, se borneraient à la feuilleter, la survoler : " j'ai eu la sensation que cela devra paraître terriblement long et peu divertissant pour les lecteurs non spécialistes (et ce seront les 9/10) et j'ai toujours la crainte que certaines catégories de lecteurs, les hygiénistes, ingénieurs, etc., ne ferment notre livre après avoir parcouru les chapitres initiaux ”, " $j$ 'ai fait lire le texte des 5 ou 6 premières feuilles, soit tirées, soit en épreuves, à quelques personnes sûres et nous voulant du bien. Hé bien, je constate, non sans terreur, une impression générale: on trouve les débuts du livre, spécialement pour Han, horriblement longs, fastidieux et peu intéressants pour qui n'est pas spécialiste ". Il est vrai que la place consacrée à la grotte de Han-sur-Lesse est prépondérante dans l'ouvrage.

La seul remède que Van-den-Broeck avait trouvé pour pallier ces sérieux inconvénients était d'établir des tables des matières et des répertoires divers, en début d'ouvrage si possible ; soit, un épaississement supplémentaire du livre et, au final, un embrouillamini inextricable !

C'est sans nul doute l'écueil majeur de ce livre,bien qu'il soit fabuleusement documenté et qu'il révèle surabondamment la grande science de ses signataires, surtout d'Ernest Van-den-Broeck, supérieurement compétent. Mais, comme il est trop fouillé, personne ne l'a lu ; peu de gens d'ailleurs avouent l'avoir parcouru en entier, les spéléologues Belges y compris!

$\mathrm{Si}$, sur le fond, sa documentation et sa structure en font l'ouvrage le plus complet et de loin le plus scientifique auquel Martel ait participé, sa rédaction compliquée et les interminables longueurs en font l'exacte antithèse de son œuvre personnelle où toujours rayonna sa pensée logique,et surtout concise.

Il n'est pas du tout sûr que le prestige de Martel ait grandi d'avoir été associé à cette œuvre. Mais il est certain que, grâce à la science d'Ernest Van-den-Broeck à laquelle il s'est nourri durant plus de douze années, Martel s'est intellectuellement enrichi considérablement dans un domaine où, enfait, il n'excellait pas, en dépit du fait qu'il avait fréquemment excursionné avec son beau-frère Louis de Launay, qui était l'une des sommités mondiales de la discipline : la géologie, que lui même avait appliquée aux cavernes.

En plus de sa notoriété internationale qu'il avait sans nul doute voulu asseoir durablement en devenant coauteur du "Livre ", la raison cachée qui pourrait expliquer que Martelait enduré autant d'années le comportement usant de Van-den-Brock est qu'il était habité de la quête personnelle inavouable suivante : acquérir enfin, peu à peu, des bases solides auprès d'un collègue expert en la matière et enclin à les lui 
fournir.

L'enseignement de ce géologue éclairé, doublé d'un spéléologue chevronné, a sans nul doute été profitable à Martel, qui en a retiré des notions nouvelles pour lui et qui a su rectifier peu à peu maintes de ses théories inexactes, ou proprement aventureuses.

C'est ainsi que, par exemple, il a fait amende honorable de ses affirmations péremptoires qui déclaraient inaptes à la consommation humaine toutes les émergences karstiques : certaines sources en terrain calcaire de Belgique (et partant, d'ailleurs) peuvent être excellentes, car filtrées par des remplissages sableux (sources de Modave, calcaires de Dinant).

Dès 1898, Van-den-Broeck lui faisait comprendre que l'action chimique était prépondérante sur l'action mécanique dans la genèse des cavernes, en règle générale, contrairement à ce que l'étude de Bramabiau lui avait enseigné empiriquement.

Martel a aussi pu étudier de nombreux recoupements souterrains de méandres (Ourthe, Lesse, etc.) qui semblent abonder en Belgique, et demeurent rares en France.

Grâce à la disposition spéciale du réseau de Rochefort,caverne percée de cheminées communiquant avec la surface, il acquit la preuve (lui semblait-il) que des abîmes pouvaient bien être à l'origine du creusement de cavités subhorizontales, faits qui, jusquelà, n'étaient que théoriques dans son œuvre personnelle.

Les Abannets de Nismes, à Couvins, et leurs remplissages gréseux, lui permirent d'affirmer l'existence de paléo-karsts en Belgique, de mêmes types que les poches àphosphorites tertiaires du Quercy français.

Mais, selon moi, l'enseignement majeur de la longue préparation de cet ouvrage est qu'elle a initié Martel à la patience et la résignation. Lui, si ordinairement si bouillant et toujours prêt à en découdre, a appris à ronger son frein. Il a trouvé en Van-den-Broeck un maître "es-polémique " contre qui il ne pouvait mais. Ce qui est très surprenant, c'est que Martel n'ait pas rompu ses relations avec lui dès la première altercation écrite, comme c'était son habitude, et comme cela fut ou allait être le cas ex abrupto avec le biologiste Armand Viré, avec le géologue Lugeon lors de l'affaire du barrage de Génissiat, avec le préhistorien Emile Cartailhac, avec Robert de Joly, etc.

Voici, à titre d'exemple, le genre d'écrit“"sympathique " que lui envoyait, de temps à autre, Van-den-Broeck: “ j'espère bien que vous n'allez plus continuerà déflorer le livre en utilisant ces données pour d'autres publications? J'avoue franchement $n$ 'avoir pas été ravi en trouvant dans les $C$. R. la primeur, sous votre nom personnel, de l'exposé des origines et de l'histoire de la grotte de Rochefort,annoncés dans le livre comme une thèse nouvelle appartenant au livre.Devant paraître dans 5 à 6 mois le livre ne fera plus que répéter ce que Martel aura dit en juin et dont la priorité est ainsi enlevée à l'œuvre commune. J'avais pensé que contribuant ici, nous deux, très exclusivement à la mise au point matérielle de la dite ceuvre commune, notre apport scientifique pouvait légitimement être et rester acquis à l'auvre dans son intégrité. Si un motif quelconque et ignoré de nous devait faire presser la publication d'un des points d'un patrimoine scientifique du livre, ce devrait être sous nos trois noms réunis en toute équité. Je vous dis franchement ma manière de voir, et ma surprise! surtout après vos légitimes récriminations vis à vis d'un confrère en spéléologie (nota: il voulait parler de Viré). Ne faisons pas aux autres ce que nous ne voulons pas qu'on 
nous fasse à nous même. Voilà le proverbe qui me revient à l'esprit. Sans rancune et bien à vous ".

Il faut croire que Martel avait, à cette date (1905),spécialement besoin que ce grand ouvrage puisse paraître, pour ne pas répliquer de façon cinglante à son correspondant et se fâcher irrémédiablement avec lui. En effet, les courriers qui suivent, s'ils évoquent encore cette affaire de publication, reprennent un ton plus affable... jusqu'à la prochaine algarade !

La seule vrai exception à cette retenue de Martel a été, en mars 1905, son refus catégorique de voir son ennemi Armand Viré associé, de près comme de loin, au livre commun. Van-den-Broeck voulait en faire le biospéologue spécialiste de la Belgique : par sa pression, Martel a réussi à le faire écarter, au grand dam de Vanden-Broeck qui, pour plaire à son correspondant parisien, a, peu à peu, abondé dans son sens. Une annotation de Martel, sur une lettre, précise : “à fond contreViré, dont je ne veux pas ". La diatribe de Martel contre Viré atteignait 16 pages !

Van-den-Broeck lui a répondu une lettre admirablement bien tournée, dont on me permettra de citer les meilleurs passages : "vous avez dî m'écrire sous l'influence énervante d'une tension électrique orageuse, car votre lettre m'a réellement épouvanté et fait quelque peine en montrant combien vous êtes surexcité envers votre ingrat ancien collaborateur et ami(...). Si j'en juge par votre lettre, si exaltée, et d'une mise au point si inexacte pour apprécier les motifs et origine de la rédaction qui vous a si fort offusqué, vous devez être prompt à vous exagérer bien des choses... et cela en explique peut-être bien d'autres, où les torts de vos adversaires sont peutêtre moindres que vous ne le pensez!Laissons toujours une large part aux malentendus. Je puis en tous cas vous affirmer que dans le cas présent ce pauvre Viré est bien loin de se douter qu'on puisse raisonnablement l'accuser de profiter de notre cuvre pour" demander sa part du gâteau " ! ou à être admis à un titre quelconque “ dans notre trio ”. Mais je me demande assez piteusement par qui maintenant nous allons, en Belgique, faire déterminer nos petites bêtes... car je n'oserai plus jamais ni prononcer le nom de M. Viré ni lui envoyer ou faire envoyer quoi que ce soit, de peur des foudres vengeresses du chatouilleux ami Martel!! Et j'ai beau me mettre Martel en tête, je ne vois pas qui va déterminer notre petit monde souterrain!" (lettre du 06 juin 1905).

N'importe qui d'autre ayant écrit cela à Martel se serait vu banni pour toujours du cercle de ses correspondants ! Mais Martel avait besoin que le livre sur la Belgique paraisse...

Et puis, il y avait la " carotte" : une promesse de distinction royale que Van-denBroeck assurait pouvoir lui faire obtenir : soit l'ordre de Léopold, soit un prix spécial d'hydrogéologie fondé par le Roi lui-même. Van-den-Broeck avait promis de remettre à ce monarque, au nom de Martel, un exemplaire spécialement relié des" Abîmes ", pour attirer son attention. De plus, il disait : “ j'ai fait intervenir, directement sous votre nom, un message utile, relatif au Roi. C'est un premier jalon que nous jetons ainsi pour vous amener la faveur royale et d'ailleurs cet hommage au Roi est absolument mérité. S. M. Léopold acréé, il y a 16 à 20 ans, un prix de 25.000 pour l'auteur du meilleur travail conduisant à l'alimentation en eau potable la Belgique par les eaux en réserve dans les terrains de la haute Belgique " (Lettre du 2 juillet 1905). "Pour ce qui concerne les démarches à faire pour votre nomination dans l'ordre de Léopold à l'occasion de l'apparition du livre, vous n'en avez. 
absolument aucune à faire; nous nous chargerons de cela ici " (25 avril 1907).

Il n'a pas été possible de savoir si Martel a finalement bénéficié de la moindre gratification royale. L'on sait seulement qu'en février 1906 il avait été promu à la dignité de Membre d'Honneur de la Société Belge de Géologie.

Quant à ce que pensait finalement Martel du long travail fait en collaboration, une ligne le résume, dans une lettre (17/09/1934) à Max COSYNS, spéléologue belge : il qualifiait “Les cavernes et les rivières souterraines de la Belgique " “d'indigeste et trop gros ouvrage”!

C'est bien ce que, personnellement, j'ai ressenti à sa très difficile lecture.

Edouard-Alfred Martel a écrit 24 ouvrages de librairie, dont deux seulement en collaboration avec d'autres auteurs. La gestation de l'ouvrage sur la Belgique, qui est, de très loin, le plus épais de toute sa phénoménale production, a été pénible, une véritable épreuve pour Martel qui ne s'est plus jamais risqué à recourir à une association pour ses volumes ultérieurs : il a été, comme on dit," vacciné " à tout jamais.

Mais, de ses tourments, des difficultés presque insurmontables auxquelles il a dû faire face, jamais il n'en a rien dit dans aucune publication - que je sache — ni dans aucune autre circonstance. Là, comme souvent, c'est dans la confidentialité de correspondances que la vérité a été débusquée. La conservation et la transmission jusqu'à nous, spéléologues de l'aube du troisième millénaire, d'un aussi épais et riche dossier entièrement consacré à cette œuvre gigantesque est une chance unique pour l'histoire de la spéléologie européenne.

La “ bible" des spéléologues de Belgique n'aurait jamais été écrite telle quelle sans l'opiniâtreté d'Ernest Van-den-Broeck, ni sans ses excès et défauts non plus ; elle n'aurait jamais vu le jour si les nerfs de Edouard-Alfred Martel avait " craqué ", s'il n'avait passu habilement résister en jeune maître face à deux disciples dont un était boulimique de gloire.

Remises en lumière après un siècle d'oubli environ,ces archives nous ont permis de fouiller dans l'intimité des grands pionniers de la spéléologie européenne, dont Martel, lesquels nous sont apparus sous leurs vrais visages d'hommes à qui nous ressemblons tant, et que, pourtant, nous avons portés au rang de demi-dieux.

Si l'ouvrage sur “ Les Cavernes et les Rivières souterraines de la Belgique "n'avait pas été conçu par des coauteurs, dont Martel, nous n'aurions jamais su comment ce dernier travaillait à l'élaboration de ses livres, lui qui communiquait peu avec ses éditeurs, sauf avec le millavois Jules Artières. La correspondance qui s'est obligatoirement installée entre ces collaborateurs étrangers, parlant la même langue mais habitant loin les uns des autres, est donc précieuse pour les historiens de la spéléologie... lesquels peuvent aussi se réjouir que Van-den-Broeck détestait le téléphone !

Que dire de ce grand ouvrage édité en deux tomes ?

C'est bien sûr une mine de renseignements et un véritable traité de géologie appliquée aux cavernes, le premier du genre sans aucun doute. Le premier tome, spécialement "spéléologique ", est consacré à l'hydrologie du Dévonien du Bassin de Dinant ; le deuxième tome, plus théorique, est dévolu àl'hydrologie pratique du Carbonifère de cette même région.

Disserter d'avantage sur cette fameuse publication reviendrait à vouloir imiter le trop prolixe Ernest Van-den-Broeck...

Les dessous de la genèse de l'une des bibles de la spéléologie européenne méritaient d'être présentés dans une revue internationale, même résumés. Au moins une fois... 


\section{REMERCIEMENTS ET SOUHAITS}

Je tiens à remercier chaleureusement mon ami Louis Renouard,arrière petit-fils du géologue Louis de Launay (beau-frère de Martel), pour m'avoir communiqué en prêt de longue durée cet épais dossier sur la Belgique souterraine d'autrefois. Je sais gré aussi à sa cousine, Madame Nicole Legrand (petite-nièce de Martel),d'avoir tout fait pour que ces précieux documents, qu'elle n'a retrouvés qu'en 1998, puissent être étudiés à loisir.

Il ne me reste plus qu'à espérer qu'après avoi rextrait dans ce présent article la quintessence de cette correspondance fabuleuse, l'Association Edouard-Alfred Martel aura la possibilité matérielle, d'ici à quelques années, d'en publier le contenu intégral.

Et je souhaite d'apprendre un jour que le versant proprement" martélien " de cette correspondance aura été découvert quelque part en Belgique. Appel est donc fait en ce sens aux érudits spéléologues belges amateurs de leur histoire.

\section{ECRITS CHRONOLOGIQUES DE MARTEL SUR LA BELGIQUE}

1) LAUNAY (Louis de)\& MARTEL (Edouard-Alfred) -1890- Note sur quelques questions relatives à la géologie des grottes et des eaux souterraines(Padirac, Han-sur-Lesse, etc.) in Bulletin de la Société Géologique de France, t.XIX, 1890-1891,p.142-165 ; tiré à part, Lille, imp. Le Bigot Frères, 1890,extrait à pagination continue

2) MARTEL (EdouardAlfred) -1894- Les Abîmes. Les eaux souterraines, les cavernes, les sources, la spelaeologie. Explorations souterraines effectuées de 1888 à 1893 en France, Belgique, Autriche et Grèce Paris, imp. CharlesDelagrave, $580 \mathrm{p}$.

3) MARTEL (EdouardAlfred) -1896- ...conférence avec projections photographiques sur les résultats géologiques de ses explorations souterraines en France, Belgique, Autriche, Grèce, Angleterre et Irlande, de 1888 à 1895... in C.R. des Séances de la Société Géologique de France, n² , séance du 24 février 1896, p.XXXV-XXXVIII

4) MARTEL(Edouard Alfred) -1896- Explorations souterraines. M. E.-A.MARTEL fait une conférence avec projections photographiques sur les résultats géologiques de ses explorations souterraines en France, Belgique, Autriche,Grèce, Angleterre et Irlande, de 1888 à 1895 in Bulletin dela Société Géologique de France, troisième série, tome 24,n² ${ }^{\circ}$, mars, séance du 24 février 1896, p.87-89

5) MARTEL (Edouard Alfred) -1896- Les grottes de Couvin (Belgique) et le contournement des siphons in Spelunca, Bulletin Société Spéléologie, 2ème année, tome 2, n 8, octobre-décembre 1896 , p. $140-142$

6) MARTEL(Edouard Alfred) - 1898- Nouvelles observations dans la grotte et la rivière de Han-surLesse (Belgique) in Comptes Rendus del'Académie des Sciences, tome CXXVII, séance du 24 octobre 1898, p.641-645 
7) MARTEL(Edouard Alfred) \& VAN DEN BROECK (Ernest) -1898- Nouvelles recherches et constatations à Han-sur-Lesse in Bulletin de la Société Belge de Géologie, séance du 25 octobre 1898, t.XII, p.157-176 ; tiré à part

8) MARTEL(Edouard Alfred) -1899- La grotte de Han (Belgique) in Revue La Nature, $I, n^{\circ} 1341,4$ février 1899, p.151-155 - II,n¹343, 18 février 1899, p.182-186

9) MARTEL(Edouard Alfred) \& VAN DEN BROECK (Ernest) -1902- "La Lesse souterraine" in Le Mouvement Géographique, n²1, 25 mai 1902,p.251-252

10) MARTEL(Edouard Alfred) -1903- Etablir, au point de vue des exigences del'hygiène, les conditions que doivent remplir les eaux issues des terrains calcaires in Congrès International d'Hygiène et de Démographie, XIème session,3ème section (Technologie sanitaire), Bruxelles, 2-8 septembre, 12p.

11) MARTEL(Edouard Alfred) -1905- Sur la formation de la grotte de Rochefort (Belgique) in Comptes Rendus de l'Académie des Sciences, séance du 19 juin 1905

12) MARTEL(Edouard Alfred) -1905- La grotte de Han sur Lesse.Belgique in Rev. Le Tour de France, Album et Guide du Touriste en France, Colonies Françaises et pays de langue française, $\mathrm{n}^{\circ} 19$, 1er juillet 1905, p. $13-16$

13) MARTEL(Edouard Alfred) \& VAN DEN BROECK (Ernest) -1906- Sur les Abannets de Nismes (Belgique) in Comptes Rendus de l'Académiedes Sciences, séance du 14 mai 1906

14) MARTEL(Edouard Alfred) \& VAN DEN BROECK (Ernest) -1906- Sur les Abannets de Nismes (Belgique) in Bulletin de la Société Belgede Géologie, t.XX, 1906, p.3-5

15) MARTEL(Edouard Alfred) -1909- Les cavernes et les rivières souterraines de la Belgique in Revue Ciel et Terre, t.30, ler novembre 1909, p.397-407

16) MARTEL(Edouard-Alfred), RAHIR (Edmond) \& VAN DEN BROECK (Ernest) -1909- Les cavernes et les rivières souterraines de la Belgique in Revue Ciel et Terre, t.30, I, 16 novembre 1909, p.421433 et II, 11,décembre, p.449-458

17) MARTEL(Edouard-Alfred) \& VAN DEN BROECK (Ernest) -1910- Sur les conditions de filtrage efficace des eaux souterraines dans certaines formations calcaires in Comptes Rendus de l'Académie des Sciences, séance du 19 septembre1910

18) MARTEL(Edouard-Alfred) \& VAN DEN BROECK (Ernest) -1910- Hydrologie. Sur les conditions de filtrage efficace des eaux souterraines dans certaines formations calcaires in Bulletin de la Société Belge de Géologie, t.XXIV, 1910,p.411-413

19) MARTEL (Edouard-Alfred), RAHIR (Edmond) \& VAN DEN BROECK (Ernest)-1910- Les cavernes et les rivières souterraines de la Belgique vol. Bruxelles, Lamertin, 1910 ; t.I : LES CALCAIRES DEVONIENS DU BASSIN DE DINANT, 12-XXIII - 786 - XL p.t.II : LES CALCAIRES CARBONIFÉRIENS DU BASSIN DE DINANT, p.787 à 1592 - 92 - LXVI p. 


\section{BIBLIOGRAPHIE EXHAUSTIVE RELATIVE À MARTEL ET LA BELGIQUE (PAR ORDRE CHRONOLOGIQUE)}

T -1898- Revue scientifique in Journal Ixelles-Canton,Organe de la Démocratie Libérale du canton d'Ixelles et des environs, $6^{\text {ème }}$ année, $n^{\circ} 18 \mathrm{du} 1^{\mathrm{er}}$ mai, $2^{\mathrm{ème}} \mathrm{p}$. (au sujet de deux conférences données par MARTEL, le 23 et 25 avril 1898, à la Société de Géographie de Belgique et àl a Société de Géologie de Bruxelles, sur " L'exploration scientifique des cavernes, de la spéléologie et des résultats de dix années de recherches dans les grottes, les abîmes, etc. de l'Europe ")

XXX -1898- Non titré in Journal L'Etoile Belge (journal de Belgique) du samedi 24septembre, p.3, rubrique "Chronique de laville" (au sujet de la venue de MARTEL à la grotte de Han-sur-Lesse en septembre 1898)

F. F. -1898- Exploration de la Lesse in supplément littéraire du Journal L'Indépendance belge(journal de Belgique) du dimanche 25 septembre (au sujet de lavenue de MARTEL à la grotte de Han-sur-Lesse en septembre 1898)

XXX -1898- Non titré in Journal La Gazette(journal de Belgique) du dimanche 25 septembre (au sujet de la venue de MARTEL à la grotte de Han-sur-Lesse en septembre 1898)

XXX -1898- La grotte de Han in Journal La Meuse(Belgique) du 07 octobre (au sujet de la venue de MARTEL à la grotte de Han-sur-Lesse en septembre 1898)

G. H. -1898- Académie des Sciences. Séance du 24octobre 1898... Géologie. - Nouvelles observations dans la grotte et la rivière souterraine de Han-sur-Lesse (Belgique). Note de M.MARTEL in Journal Le Génie Civil (Belgique), novembre (au sujet d'un article de MARTEL relatif à la grotte de Han-surLesse, septembre 1898)

XXX -1898- Exploration de la grotte de Han-sur-Lesse(Belgique) in Journal Le Cosmos du 05 novembre (au sujet de la venue de MARTEL à la grotte de Han-sur-Lesse en septembre1898)

XXX -1898-Spéloeologie in Revue Scientifique du 05 novembre (au sujet de la venue de MARTEL à la grotte de Han-sur-Lesse du 18 au 20 septembre 1898)

KIMANKA-DISSO -1898- Chronique scientifique - Petite revue d'astronomie météorologique et physique du globe in Journal L'Indépendance Belge du19 décembre (au sujet d'un article de MARTEL sur la grotte de Han-sur-Lesse, septembre 1898)

A. V. W. -1899- A) Explorations nouvelles dans la grotte de Han (Ciel et Terre, $1^{\circ}$ année, 1898 , p.468-473 ; plan de la Lesse souterraine par E.A. MARTEL et VAN DEN BROECK). - B) Nouvelles observations sur la grotte et la rivière souterraine de Han-sur-Lesse (Belgique) (C. R. de l'Académie des Acad. Sc., CXXVII,1898, p.641-645, 1 croquis) in Annales de Géographie, 15septembre

ARDENNE (Jean d') signé J. d'A. -1902- L'Homme des Abîmes in Journal La Chronique, Gazette Quotidienne, (journal de Belgique), 35ème année, $\mathrm{n}^{\circ} 137$ du jeudi 22 mai, p.2 (sur Edouard-Alfred MARTEL, au sujet d'une conférence donnée le mardi 20 mai 1902 à Bruxelles, sous les auspices de la Société Belge de Géologie) 
ARDENNE (Jean d') -1902-Notes d'un vagabond in Journal La Chronique, Gazette Quotidienne, (journal de Belgique), 35ème année, $n^{\circ}$ du mercredi 04 juin (sur Edouard-Alfred MARTEL)

XXX -1902- Chronique de la ville in Journal L'Etoile Belge du jeudi 05juin, p.3 (au sujet d'une campagne de onze jours d'explorations spéléologiques, par MARTEL, VAN DEN BROECK, RAHIR, etc., en vue d'une révision des cavernes belges)

XXX -1902- Huy.Au Trou-Manto in Journal La Meuse (Belgique), du dimanche 08 juin (au sujet de l'exploration, le 25 mai 1902, de la grotte du trou-Manto, par MARTEL et associés)

ARDENNE (Jean d') -1902-Notes d'un vagabond in Journal La Chronique, GazetteQuotidienne, (journal de Belgique), 35ème année, dimanche 08 juin (sur Edouard-Alfred MARTEL, au sujet de l'exploration de la grotte de Rémouchamps et d'un gouffre de $52 \mathrm{~m}$ de profondeur)

ARDENNE (Jean d') -1902- Notes d'un vagabond in Journal La Chronique, Gazette Quotidienne,(journal de Belgique), 35 ème année, ${ }^{\circ} 157$ du mercredi 11 juin, p.1 ; extrait titré A travers les journaux. Le Trou-Manto repris in Journal la Meuse du vendredi 13 juin 1902 (sur Edouard-AlfredMARTEL, au sujet de l'exploration de la grotte de Lovegnée, ou Trou Manto, en compagnie de Victor MARTIN, et des grottes du Pré-au-Tonneau, du Trou-Maulin, d'Eprave, de Rochefort, de Han, Trou de l'Abîme à Couvin, etc.)

GEORGERRAND -1902- Sous terre in Journal Encyclopédie du Soir (Belgique) du 16 juillet (sur Edouard-Alfred MARTEL et ses explorations de chantoirs près de Rémouchamps, chantoirs de Béton-Ry, Trou du Coq, Grandchamp, Adseux, Comblain-aux-Pont, sur la grotte de Tilff qu'il n'a pu visiter à cause d'une crue, sur l'abîme de Ramioul près d'Engis, sur le Trou-Manto, sur la grotte d'Eprave - la Lomme sourterraine - sur le Trou-du-Pré-au-Tonneau, sur le Trou Maulin, sur la grotte de Rochefort, sur la grotte de Han-sur-Lesse,sur le Trou-Madame, le trou Picot, le trou Sinsin, sur l'abîme del'Eau Noire = Trou de l'Abîme à Couvin)

XXX -1905- Non titré in Journal L'Indépendance Belge du 24 juin (Compte rendu bibliographique d'un article de MARTEL paru dans les $C$. $R$. del'Académie des Sciences, au sujet de la grotte de Rochefort et sa formation supposée en liaison avec quatre gouffres absorbants)

A. B. -1905- Sociétés Savantes et Industrielles. Académie des Sciences. Séance du 19 juin1905 in Journal Le Génie Civil du 01 juillet (Compte rendu bibliographique d'un article de MARTEL paru dans les $C$. R. del'Académie des Sciences, au sujet de la grotte de Rochefort et sa formation supposée en liaison avec quatre gouffres absorbants)

XXX -1905- A la Sociétéde Géographie in Le Journal de Bruxelles (Belgique) du 06octobre ; reprise (non titrée) in Journal L'Indépendance Belge du 06 octobre 1905 ; reprise (non titrée) in Journal L'Etoile Belge du 06 octobre1905 (au sujet d'une conférence à venir de Edouard-Alfred MARTEL à la Société Royale belge de Géographie,pour le 19 octobre)

XXX -1905- Découverte archéologique in Journal le XX Siècle (Belgique) du mardi24 (au sujet de la découverte de cinq sépultures préhistoriques dans le site de Furfooz, et notamment dans le Trou de la Mâchoire, ainsi que sur la visite de la grotte de Montfort, de la grotte de Bouvignes, de la grotte des fonds de Leffe et de la nouvelle grotte de Dinant) 
XXX -1909- Les cavernes etrivières souterraines de la Belgique, étudiées spécialement dans leurs rapports avec l'hydrologie des calcaires et la question des eaux potables, par $E$. Van denbroeck, $E$.

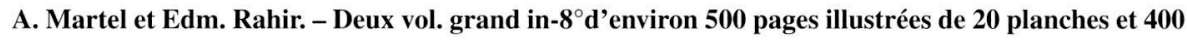
gravures (Berqueman, 12, rue du Boulet,Bruxelles) in Rev. Les Livres Nouveaux, Avignon, juillet, rubrique" V. - Géographie. -Voyages", p.165-167 (recension de l'ouvrage Les cavernes et les rivièressouterraines de la Belgique)

XXX -1909- Les Cavernes de la Belgique in Journal La Gazette (Belgique) du lundi 20 décembre(recension de l'ouvrage Les cavernes et les rivières souterraines de la Belgique)

RAHIR (Edmond) -1909- Merveilles souterraines de la Belgique Bruxelles, Lebègue éditeur, TouringClub de Belgique, $237 \mathrm{p}$.

ARDENNE (Jean d') -1910- Chronique bibliographique. Sous terre in Journal La Chronique,Gazette Quotidienne, (journal de Belgique), 42èmeannée, numéro du jeudi 27 janvier (recension de d'Edmond RAHIR Les merveilles souterraines de la Belgique)

ARDENNE (Jean d') -1910- Cavernes et eaux souterraines in Journal La Chronique, GazetteQuotidienne, (journal de Belgique), 43ème année,numéro du lundi 28 février, p.1 (recension de Les cavernes et les rivières souterraines de la Belgique)

POSKIN (Dr A.) -1910- Chantoirs, abîmes, grottes,cavernes et eaux souterraines in Journal La Meuse, (journal de Belgique), du samedi 26 mars (recension de l'ouvrage Les cavernes et les rivières souterraines de la Belgique)

XXX -1910- Les livres in Journal L'Etoile Belge du dimanche 27 mars (recension de l'ouvrage Les cavernes et les rivières souterraines de la Belgique)

L. -1910- Les cavernes et les rivières souterraines de la Belgique étudiées spécialement dans leurs rapports avec l'hydrologie des calcaires et avec la question des eaux potables Journal L'Indépendance Belge du lundi 29 mars (recension de Les cavernes et les rivières souterraines de la Belgique)

XXX -1910- Spéléologie et hydrologie. Les calcaires filtrants et les rivières souterraines filtrées in Rev.L'Eau, ${ }^{\circ} 4$ du 15 avril, p.39-42 (recension de l'ouvrageLes cavernes et les rivières souterraines de la Belgique)

XXX -1910- Spéléologie et hydrologie. Les calcaires filtrants et les rivières souterraines filtrées in Rev.L'Eau, ${ }^{\circ} 4$ du 15 avril, p.39-42 (recension de l'ouvrage Les cavernes et les rivières souterraines de la Belgique)

P. L. -1910- Les cavernes et les rivières souterraines de la Belgique, par E. VAN DEN BROECK, E.A.MARTEL et EDM RAHIR. 2 vol. grand in $-8^{\circ}$ de $12+$ XXIII + $1592+92+$ XL + XLVIII pp. (total : 1807 pp.), avec 26 planches en couleurs et 435 simili-gravures, cartes,plans et coupes, H. Lamertin, Bruxelles. Prix : 25 francs in Rev.Scientifique, juin (recension de l'ouvrage Les cavernes et les rivières souterraines de la Belgique) 
ZADIG -1910- Eaux potables in Journal de Bruxelles (Belgique) du mercredi 13juillet (recension de l'ouvrage Les cavernes et les rivières souterraines de la Belgique)

XXX -1910- Filtrage des eaux dans les terrains calcaires in Journal Officiel de la République Française dudimanche 20 septembre, rubrique “ Académie des Sciences - Séance du 19 septembre. Présidence de M. A. Gautier "p.8072 (compte rendu d'un article à l'Académie des Sciences de MARTEL et de VAN-DENBROECK)

RUDAUX (Lucien) -1910- L'hydrologie souterraine de la Belgique ardennaise in Rev. La Géographie, tome XXI, p.601-605 (recension de l'ouvrage "Les Cavernes et les Rivières souterraines de la Belgique", de E.-A. Martel, E. Rahiret E. Van den Broeck)

(Auteur inconnu) -1910- (TITRE INCONNU) in Bull. American Geographical Society, tome 42, n4,p.292-294 (recension de “Les Cavernes et les Rivières souterraines de la Belgique; référence de secondemain due à Trévor-R. Shaw)

XXX -1956- Hommage au fondateur de la spéléologie in Journal Le Matin, Anvers(Belgique) du 2 mars

\section{BIBLIOGRAPHIE RESSOURCE}

ARCHIVES DEPARTEMENTALES DE LA LOZERE \& ASSOCIATION EDOUARD-ALFRED-MARTEL -1999- L'homme qui voyageait pour les gouffres, Actes du Colloque Edouard-Alfred Martel tenu à Mende les 17 et 18 octobre 1997, 424 p.

ASSOCIATION EDOUARD-ALFRED MARTEL -1997- La Plume et les Gouffres, Correspondance d'Edouard-Alfred Martel(1859-1938) Saint-Georges-de-Luzençon, imp. Causses et Cévenne, 608 p. (1034 lettres dont 425 de la main de Martel ; 30 pages de répertoire chronologique sur sa vie)

CASTERET (Norbert) -1943- E.-A. Martel. Explorateur du Monde Souterrain Paris, Gallimard, 234 p.

CHABERT (Claude) \& COURVAL (Michel de) -1971- E.-A. Martel1859-1938. Bibliographie Autun, imp. Marcellin et Cie, 103 p. (922références)

FOUNTES (Jacques) -1997- Qui êtes-vous, Monsieur...Edouard-Alfred MARTEL Marvejols, imp. des 4, Edité par leConseil Général de la Lozère à l'occasion de l’Année Martel- 1997; mai, 34 p.

MARTEL (Edouard-Alfred) (signé “Gabriel GAUPILLAT”) -1891- En ballon libre ! in Annuaire du Club Alpin Français, 18ème année, p.403-422

PARC NATIONAL DES CEVENNES -1998- Sur les traces d'Edouard Alfred Martel... Quelle nouvelle politique pour les espaces protégés? Evolution des regards, solidarités et coopérations sur nos territoires, Actes du Colloque de Florac des 1er, 2 et 3 mai 1997, Parc National des Cévennes/Réserve de biosphère des Cévennes, Horizons Parcs Nationaux, 119 p. 